Conf-9009235--3

UCRL-JC- -104971

DE91 002673

\title{
X-Ray Holography at Lawrence Livermore National Laboratory
}

\author{
J. Trebes, C. Annese, D. Birdsall, J. Brase, J. Gray, S. Lane, \\ R. London, D. Matthews, D. Peters, D. Pinkel, G. Stone, D. Rapp, \\ M. Rosen, U. Weier and T. Yorkey
}

This paper was prepared for submittal for the Proceedings of the International X-Ray Microscopy Colloquium

London, England, Septemer 3-7, 1990

October 11, 1990

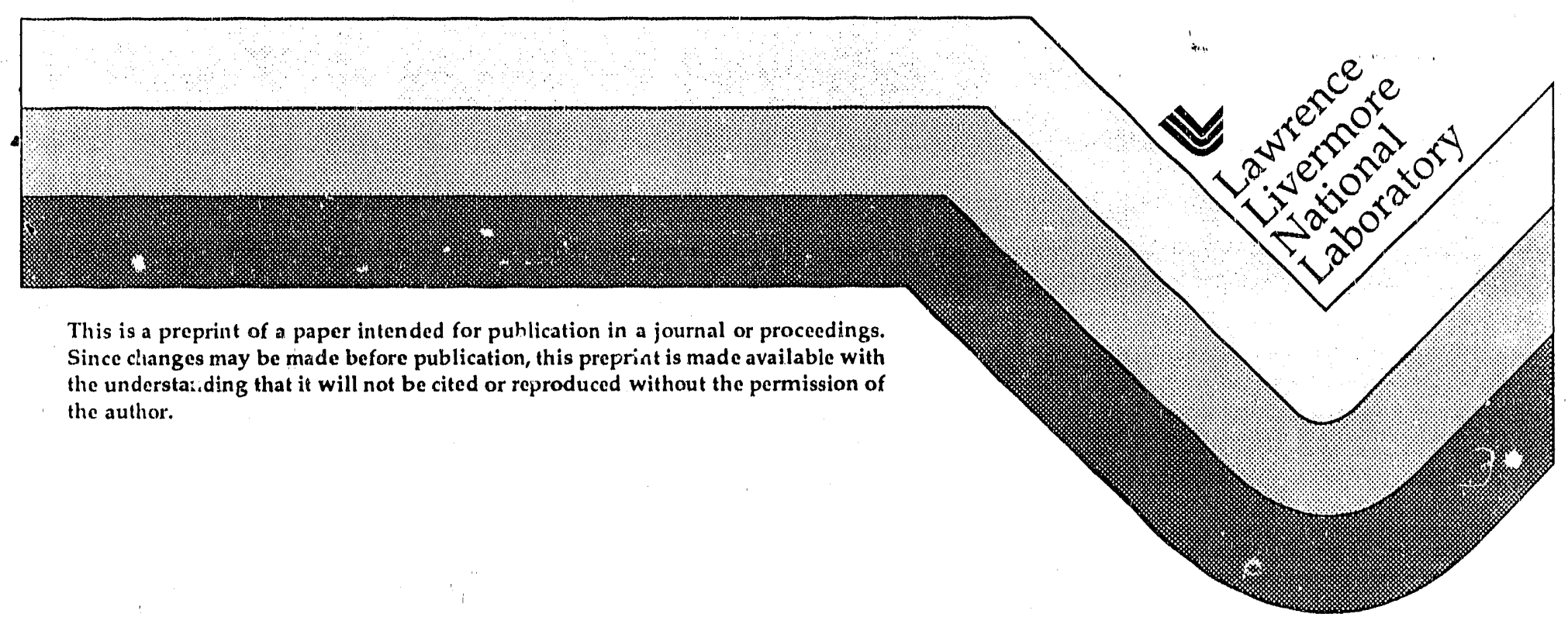


DISCI.AIMER

This document was prepared as an account of work sponsored by an agency of the United States Government. Nelther the United States Government nor the University of Callfornia nor any of their employees, makes any warranty, express or Implied, or assumes any legal liability or responsibility for the accuracy, completeness, or usefulness of any information, apparatus, producl, or process disclosed, or represents that its use would not infringe privately owned rights. Reference herein to any speciflc commercial products, process, or service by trade name, trademark, manufacturer, or otherwise, does not necessarily constitule or imply its endorsement, recommendation, or favoring by the I/nited States Government or the University of California. The views and opinions of authors expressed herein do not necessarily state or reflect those of the United States Government or the University of California, and shall not be used for advertising or product endorsement purposes. 


\title{
X-ray Holography at Lawrence Livermore National Laboratory*
}

\author{
J. Trebes, C. Annese, D. Birdsall, J. Brase, J. Gray, S. Lane, R. London, \\ D. Matthews, D. Peters, D. Pinkel, G. Stone, D. Rapp, M. Rosen, U. Weier, \\ and $T$. Yorkey
}

\author{
Lawrence Livermore National Laboratory \\ P.O. Box 808 \\ Livermore, CA 94550
}

The $\mathrm{x}$-ray holography program at the Lawrence Livermore National Laboratory has two principal goals:

1) the development of $x$-ray diffraction techniques for DNA sequence analysis and 2) the development of $x$-rav laser holography for structural analysis of intact biological cells and organelles. DNA sequence analysis will be accomplished by applying $x$-ray diffraction techniques to determine the ensemble average of the sequence of labels along the individual elements of crystalline DNA. X-ray laser holographic imaging will be accomplished by applying three dimensional $\mathrm{x}$-ray holography to elucidate the structure of few hundred angstrom objects such as $300 \AA$ chromatin fibers, nuclear pores and nucleic acid replication complexes in living cells. Existing laboratory $\mathrm{x}$-ray lasers will be utilized to produce flash $\mathrm{x}$ ray holograms of the biological structures.

\section{DNA Sequence Analysis Based on X-Ray Diffraction}

Currently DNA sequence analysis is usually accomplished using procedures based on electrophoresis techniques which are relatively siow. A potentially faster method is to use $\mathrm{x}$ ray diffraction techniques. In this approach, the target DNA sequence is amplified to pro iuce a large number of identical copies and selected sites along the DNA fragments (e.g. all bases of one type) are labeled with atoms that scatter $\mathrm{x}$-rays èfficiently (e.g. with high atomic number elements such as iodine or mercury). The labeled DNA fragnients are made linear and oriented with their long axes perpendicular to a beam of coherent X-rays. High energy x-rays with a wavelength smaller than the required resolution are used. Typically this will be approximately $1.5 \AA$. The distribution of labels along the target DNA sequence is determined by Fourier analysis of the resulting coherent $x$-ray scattering pattern. The Fourier transforms of the coherent $x$-ray scattering patterns from four samples of the same target DNA sequence gives the information needed for complete DNA sequence analysis. Each of the four samples has only one of the four types of bases labeled. The interpretation of the relative positions of the labels along the copies of the target DNA sequence is facilitated by labeling one end of each copy of the target DNA sequence with a distinctive, high atomic number label. This allows specification of the positions of the labels relative to the distinctive end label. In general, the end label will be separated from the target DNA sequence by a distance that is comparable to the length of the target DNA sequence.

Large numbers (e.g. $\sim 10^{12}-10^{14}$ copies) of essentially identical copies of the target DNA sequence are required in order to produce an interpretable diffraction pattern without significant radiation damage in the sample. These copies will be obtained either by clonal multiplication in a bacterial host, or by an in vitro amplification method such as the polymerase chain reaction (PCR). DNA base labels will be introduced by continuation of the PCR reaction in the presence of one halogenated dNTP (e.g. Iododeoxyuridine; I-dUTP). This results in the incorporation of a halogen at the position of each base of one type (e.g. all thymines will be labeled with an iodine by amplification in the presence of I-dUTP).

Labeled copies of the target DNA sequence will be arranged in a near linear form with their long axes perpendicular to the coherent X-ray beam for determination of the positions of the high atomic number labels along the target DNA sequence. Linear positioning of the fragments will be attempted several methods including parallel positioning in an electrophoretic environment, orientation in a DNA fiber, and orientation in a liquid crystal. A proof-ofprinciple experiment is under construction. This experiment will utilize a rotating anode source 
using a $\mathrm{Cu}$ target to produce $1.54 \AA \mathrm{K}$ line radiation. A pyrolytic carbon crystal is used to eliminate broadband radiation from the source. Sufficient spatial coherence is produced through the use of pinhole and placing the sample DNA far from the source. A 30 base long synthetic DNA sequence with 2 labeled bases and 1 reference label will be used. The oriented DNA sample distribution will be obtained by using either a liquid crystal or by pulling the DNA in a fiber. The diffraction pattern will be recorded on x-ray film. Computer simulations of this experiment, which include all the atoms within the DNA sequence, indicate that the labeled sequence should be clearly obtained from the predicted diffraction pattern 1 .

\section{X-Ray Laser Holography}

Much of modern biology is directed toward the analysis of the function of macromolecular structures (e.g., chromatin-enzyme replication complexes, nuclear pore structures, structures of protein complexes, elements of the apparatus, etc.) smaller than the.resolution limits of light microscopes $(\sim 2000 \AA)$. This trend should steadily increase as the international genomics effort provides genetic information about the proteins that mediate cell function. We are developing $\mathrm{x}$ ray laser holographic imaging to meet these new requirements for biological microscopy. This technique utilizes the coherent $x$-ray beam from an $x$-ray laser to produce a flash $x$-ray hologram of the object under investigation. This hologram is then numerically reconstructed to provide a three dimensional image of the object with high spatial resolution. Several aspects of this microscopic technique are being investigated inclucing: a) $x$-ray scattering physics, $b$ ) $x$ ray hologram simulation and numerical reconstructio:1, c) development of $x$-ray holography geometries, and d) x-ray laser development.

The optimal wavelength for producing an $x$-ray hologram of biological objects in water has been determined by a detailed investigation of the physics of coherent $x$-ray scattering from biological materials such as protein 2 . The optimal wavelength is the $x$-ray wavelength which maximizes the coherent $\mathrm{x}$-ray scattering and simultaneously minimizes the $\mathrm{x}$-ray absorption. This optimum is about $44 \AA$ just outside the "water window" between the carbon and oxygen $\mathrm{K}$ edges $(43.7-23 \AA)$ which was previously believed to be the optimal wavelength regime. A secondary optimum allowing more penetration is at $23 \AA$.

The damage caused by the $x$ rays that are absorbed by the object during the creation of the $\mathrm{x}$-ray hologram also has been investigated. The production of an interpretable hologram requires that sufficient photons be scattered to allow detection of approximately 10() coherently scattered photons from each object pixel. These scattered photons must be produced on submillisecond timescales to prevent biological motion and Brownian motion from compromising the resolution. For small objects $(-300 \AA)$ heat conduction is not effective on these timescales and significant heating accurs. This heating has been calculated. Achieving a spatial resolution of $300 \AA$ will result in sample heating to approximately $6000 \% \mathrm{~K}$. This will cause a rapid hydrodynamic expansion which compromises the resolution as well as destruction of the object. If the exposure time is less than 50 picoseconds, the object pixels do not have sufficient time to expand significantly beyond their initial size. Thus, the hologram must be produced before the x-ray heating induced explosion can destroy the object. These effects force the use of pulsed $x$-ray sources such as $x$-ray lasers which have the necessary high peak powers for producing holograms on these time scales.

The $x$-ray damage problem can be significantly reduced if the biological object has structures of interest within it labeled with high x-ray scattering cross-section materials such as gold. Microspheres of gold as small as $10 \AA$ in diameter can be attached to structures within cells with techniques such as immunogold labeling. This allows specific structures to labeled and the $\mathrm{x}$-ray fluence required to image these structures can be significantly reduced. The use of $300 \AA$ gold microspheres reduces the required $x$-ray fluence by a factor of $6($ ). This results in the object reaching temperatures approximately at the boiling point. The resulting expansion is reduced allowing exposure times of a few hundred picoseconds, well within the capabilities of existing x-ray lasers. Using larger gold spheres will result in temperatures below the boiling point and in negligible expansion. In this case, the exposure time is limited by the time scales for biological motion and other x-ray sources such as synchrotrons can potentially be used.

Computer codes for the simulation of and reconstruction of $\mathrm{x}$-ray holograms have been developed. These codes are used to evaluate different holography systems and to determine their realistic performance capabilities. These codes are validated by testing their predictions 
and reconstructed images against visible light holography experiments with known objects. Simulation studies suggest that multiple-view holography will be required for analysis of the three dimensional structure of macromolecules with few hundred Angstrom resolution. Multiple views are required because the depth resolution is proportional to the square of the hologram $f$-number. Typical holography systems will have f-numbers of $\sim 5$ beciuse of the spatial distribution of the scattered $x$-rays. Thus, the depth resolution that can be achieved from a single hologram with a $44 \AA \mathrm{x}$-ray laser is $\sim 1000 \AA$. Holograms produced simultaneously at different angles can be combined to achieve both high three dimensional spatial resolution and reduced noise. This has been demonstrated in numerical simulations of $x$-ray holograms of simple protein rods and spheres in water.

We have conducted preliminary $x$-ray holography experiments using an $x$-ray laser and initiated the design of a conceptual $x$-ray holography system which is capable of producing high three dimensional spatial resolution of biological objects. In the initial x-ray holography experiments, a selenium $x$-ray laser with a wavelength of $207 \AA$ was used to make Gabor holograms of 8 micron diameter carbon fibers and 10 micron thick gold bar figures ${ }^{3}$. These experiments demonstrated that $\mathrm{x}$-ray lasers are sufficiently intense and coherent to produce holograms in exposure times of $\sim 200$ picoseconds and that multi-layer $x$-ray mirrors are sufficiently flat and smooth that they can be successfully used in phase sensitive x-ray experiments.

We have produced a conceptual design of an $\mathrm{x}$-ray laser holography system for biological applications. This system is based on Fourier transform holography and uses multiple views. The $x$-ray laser used is a nickel-like Ta laser operating at a wavelength of $44.83 \AA^{4}$. Lasers with wavelengths as short as $35.6 \AA$ have been dernonstrated. In this system different spatially coherent portions of the $x$-ray laser beam directer by multi-layer $x$-ray mirrors along a different lines of sight through the object. Adjacent to the object is a reference scatterer which consists of a gold disk or sphere. X-rays scattered from the object interfere with X-rays scattered from the reference scatterer at detectors placed along each line of sight produced by the optic. X-ray film is used as a detector, although a CCD detertor could be used. If three views are recorded, the x-ray laser needs to have an output energy' of $\sim 50$ microjoules to produce interpretable holograms on each detector. This would result in a three dimensional resolution of $300 \AA$. The object is maintained in an aqueous environment in an $\mathrm{x}$-ray transparent boron nitride holder.

1. T. Yorkey, J. Brase, J. Trebes, S. Lane and J. Gray, SPIE 1990 International Symposium on Optical and Optoelectronic Applied Science and Engineering, July 8-13, 1990, proceedings to be published.

2. R. London, M. Rosen, and J. Trebes, "Wavelength Choice for Soft X-Ray Laser Holography of Biological Sarr.ples," App. Opt.,28, 3397-3404, (1989).

3. J. Trebes, et al., "Demonstration of X-Ray Holography with an X-Ray Laser," Science, 238, 517-517, (1987).

4 B. MacGowan, et al., Phys. Rev. Lett., 65, 420, (1990).

*This work was performed under the auspices of the U.S. Department of Energy by Lawrence Livermore National Laboratory under contract number W-7405-Eng-48. 


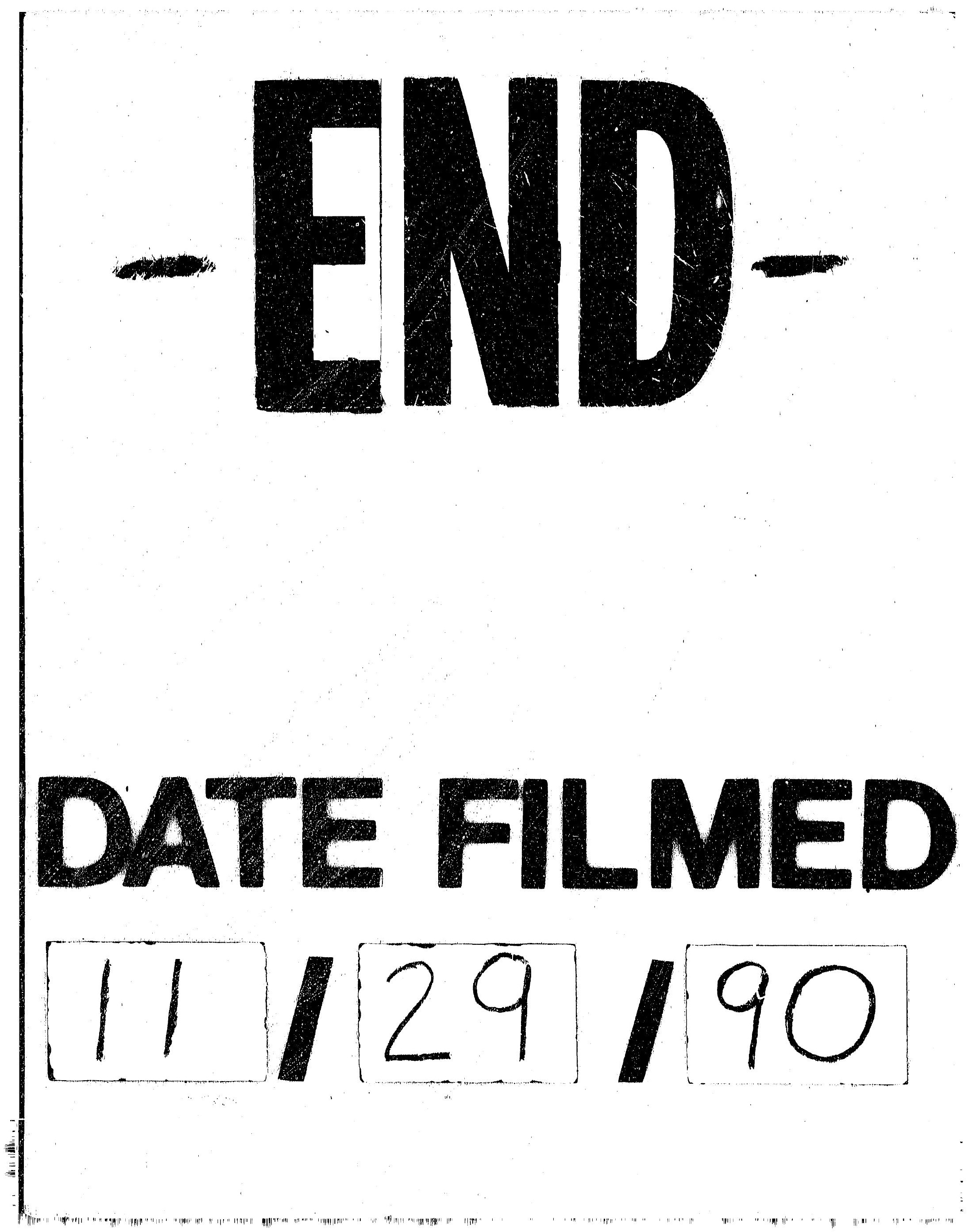




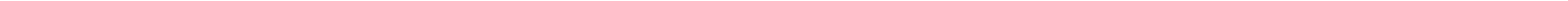

